\title{
Colon cancer in France: evidence for improvement in management and survival
}

\author{
C Faivre-Finn, A-M Bouvier-Benhamiche, J M Phelip, S Manfredi, V Dancourt, J Faivre
}

Gut 2002;51:60-64

Background: Cancer registries recording all cases diagnosed in a well defined population represent the only way to assess real changes in the management of colon cancer at the population level.

Aims: To determine trends over a 23 year period in treatment, stage at diagnosis, and prognosis of colon cancer in the Côte-d'Or region, France.

Patients: A total of 3389 patients with colon cancer diagnosed between 1976 and 1998.

See end of article for authors' affiliations Methods: Time trends in clinical presentation, surgical treatment, chemotherapy treatment, stage at diagnosis, postoperative mortality, and survival were studied. A non-conditional logistic regression was performed to obtain an odds ratio for each period adjusted for the other variables. To estimate the independent effect of the period on prognosis, a relative survival analysis was performed.

Correspondence to: C Faivre-Finn, Registre Bourguignon des Cancers Digestifs (INSERM EPI 106), Faculte de Medecine, 7 Boulevard Jeanne d'Arc, 21079 BP 87900, Dijon, France; jean.faivre@u-bourgogne.fr

Accepted for publication 23 October 2001

Results: Between 1976 and 1991, the resection rate increased from $69.3 \%$ to $91.9 \%$ and then remained stable. This increase was particularly marked in the older age group (56.4\% to $90.5 \%)$. The proportion of stage III patients treated with adjuvant chemotherapy rose from $4.1 \%$ for the 1989-1990 period to $45.7 \%$ for the $1997-1998$ period. Over the 23 years of the study the proportion of stage I and II patients increased from $39.6 \%$ to $56.6 \%$, associated with a corresponding decrease in the proportion of patients with advanced stages. Postoperative mortality decreased from $19.5 \%$ to $7.3 \%$. This led to an improvement in five year relative survival (from 33.0\% for the $1976-1979$ period to $55.3 \%$ for the 1992-1995 period).

Conclusions: Advances in the management of colon cancer have resulted in improving the prognosis of this disease. However, progress is still possible, particularly in the older age group.

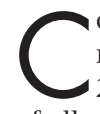
olon cancer is a major health problem. It is one of the most frequent cancers in both sexes in France, affecting of all newly diagnosed cancers.

Recent studies have demonstrated an increase in the incidence of colon cancer and this trend will continue, at least because of the increasing life expectancy. ${ }^{2}$ However, the mortality rate has remained stable over the last 20 years, suggesting an improvement in prognosis. ${ }^{1}$ Possible explanations are that both early detection and treatment of this cancer have improved. Adjuvant therapy developed in the last decade may also have had a role to play in this improvement. Data on the management and prognosis of colon cancer have mostly been provided by specialised hospital units and as such cannot be used as reference because of unavoidable selection bias. Population based studies recording all cases diagnosed in a well defined population represent the only way to assess improvement in the management of colon cancer. Some cancer registries collect detailed data on therapeutic approaches allowing clinicians to access information about changes in the management of cancer at a population level. This interest is recent and therefore there is a paucity in the literature of reports of this type. The objective of this study was to determine trends over a 23 year period in treatment, stage at diagnosis, and prognosis in a well defined French population.

\section{POPULATION AND METHODS \\ Population}

A population based cancer registry has, since 1976, recorded all digestive tract cancers occurring in the Côte-d'Or, Burgundy, France. This area has a population of 493000 according to the 1990 census. Information is regularly obtained from pathologists, hospitals (university hospitals, including the cancer centre, general hospital, and private physicians; gastroenterologists, surgeons, oncologists, and radio- therapists), and general practitioners as well as from public administration (death certificates). No cases were registered through death certificates alone but they were used to identify missing cases. Because of the involvement of the entire medical profession, we assumed that nearly all newly diagnosed cases were recorded.

Cancers were classified according to the International Classification of Diseases, 10th revision. ${ }^{3}$ A total of 3419 incident cases of colon cancer (ICD-10C18) were recorded between 1976 and 1998. Histological types including lymphomas $(n=22)$, sarcomas $(n=2)$, and carcinoids $(n=6)$ were excluded as their natural history and management are quite different to other carcinomas. A total of 3389 patients were considered for this study.

\section{Studied variables}

Available variables included sex, age at diagnosis, place of residence, date at diagnosis, histological type, location of the tumour, clinical presentation, stage at diagnosis, and surgical and chemotherapy treatment modalities. Two categories were assigned by age: those aged $<75$ years $(n=1869)$ and those $\geqslant 75$ years $(n=1520)$. Place of residence was recorded as university hospital town (Dijon, $\mathrm{n}=1462$ ), other urban area (that is, towns of more than 2000 inhabitants, $n=678$ ), and rural areas $(n=1249)$. The period of diagnosis was divided into five groups of four years and one group of three years for the last study period. As chemotherapy was an anecdotal treatment before 1989, this variable was studied using two time periods between 1989 and 1998. Location of the tumour was divided into right colon (caecum, ascending, hepatic flexure, transverse, $n=1472$ ), left colon (splenic flexure, descending, sigmoid, $n=1896)$, and unknown location $(n=21)$. Surgical procedures were divided into curative resection $(n=2452)$, palliative resection $(n=447)$, and palliative surgery with no 
Table 1 Time trends in emergency management of colon cancer between 1976 and 1998

\begin{tabular}{|c|c|c|c|c|c|c|}
\hline & $\begin{array}{l}\text { Colostomy followed by } \\
\text { resection (n (\%)) }\end{array}$ & $\begin{array}{l}\text { Colostomy alone } \\
\text { (n (\%)) }\end{array}$ & $\begin{array}{l}\text { Surgical resection } \\
\text { with stoma (n (\%)) }\end{array}$ & $\begin{array}{l}\text { Surgical resection } \\
\text { without stoma (n (\%)) }\end{array}$ & $\begin{array}{l}\text { Exploratory } \\
\text { laparotomy (n (\%)) }\end{array}$ & $\begin{array}{l}\text { No surgery } \\
(n(\%))\end{array}$ \\
\hline 1976-1979 & $5(7.3)$ & 13 (18.8) & $9(13.1)$ & $35(50.7)$ & $3(4.3)$ & $4(5.8)$ \\
\hline 1980-1983 & $12(14.8)$ & 13 (16.0) & $10(12.4)$ & 41 (50.7) & $4(4.9)$ & $1(1.2)$ \\
\hline $1984-1987$ & $11(15.9)$ & $5(7.3)$ & $12(17.4)$ & $41(59.4)$ & 0 & 0 \\
\hline 1988-1991 & $5(6.7)$ & $4(5.3)$ & $6(8.0)$ & 59 (78.7) & 0 & $1(1.3)$ \\
\hline 1992-1995 & $4(5.1)$ & $6(7.7)$ & 13 (16.7) & $52(66.6)$ & $2(2.6)$ & $1(1.3)$ \\
\hline 1996-1998 & $7(7.6)$ & $2(2.2)$ & $21(22.8)$ & $60(65.2)$ & 0 & $2(2.2)$ \\
\hline
\end{tabular}

tumour resection (that is, colostomies or explorative laparotomies, $n=208$ ). Resection was considered curative when the surgeon deemed the tumour completely removed and the distal margins were free from tumour, with no evidence of distant metastasis. Cancer extension at the time of diagnosis was classified, for resected cancers, according to the TNM classification, 1997 revision $^{4}: 566$ patients were classified as stage I, 138 stage II, 814 stage III, and 725 stage IV. Those who underwent resection but were not staged were classified as unknown $(n=11)$. Those in whom the cancer was not resected (bypass, exploratory laparotomy, no laparotomy) and with no evidence of visceral metastasis were classified as advanced stage $(n=135)$. This group of patients was analysed together with TNM stage IV. Adjuvant chemotherapy $(n=165)$ was defined as chemotherapy given after resection with curative intent for stage I, II, or III tumours. Palliative chemotherapy $(n=115)$ was defined as chemotherapy given to patients who did not undergo surgery and/or to patients with metastatic disease. Postoperative mortality was defined as death within 30 days of surgery. Complete follow up to January 2000 was obtained for $98.2 \%$ of patients.

\section{Statistical analysis}

Associations between categorial data were analysed using $\chi^{2}$ tests for heterogeneity. A non-conditional logistic regression was used to obtain odds ratios associated with the probability of tumour resection and with the probability of performance of adjuvant chemotherapy for each period adjusted for the other variables. Computations were performed using the BMDP software package. ${ }^{5}$ Relative survival rates were computed using the Relsurv 1.0 program for relative survival (Guy Hedelin, Strasbourg, France). Survival rate is defined as the ratio of the observed survival rate to the expected survival rate in that area according to sex and age group. Multivariate analysis was performed using a relative survival model with proportional hazard applied to the net mortality by interval. This model makes it possible to calculate relative risks in comparison with a baseline which is the cumulative net hazard calculated from a priori defined intervals. ${ }^{6}$ The last study period (1996-1998) has not been considered in the survival analysis because the five year follow up is not yet available for all patients.

\section{RESULTS}

\section{Clinical presentation}

Overall, $77.9 \%$ of patients presented with symptoms and $13.7 \%$ presented as an emergency (obstruction and/or perforation of the colon). The remaining $5.8 \%$ were patients diagnosed after a routine examination or faecal occult blood testing. In $2.6 \%$ of cases the type of presentation was unknown. Presentation as an emergency was influenced by age $(12.6 \%$ in the younger age group compared with $16.5 \%$ in those aged 80 years and older; $\mathrm{p}=0.003)$, by location of the tumour ( $15.0 \%$ for left colon compared with $12.1 \%$ for right colon; $\mathrm{p}=0.014)$, and by stage at diagnosis $(2.1 \%$ for stage $\mathrm{I}$, $16.3 \%$ for stage II, $18.1 \%$ for stage III, and $13.7 \%$ for advanced

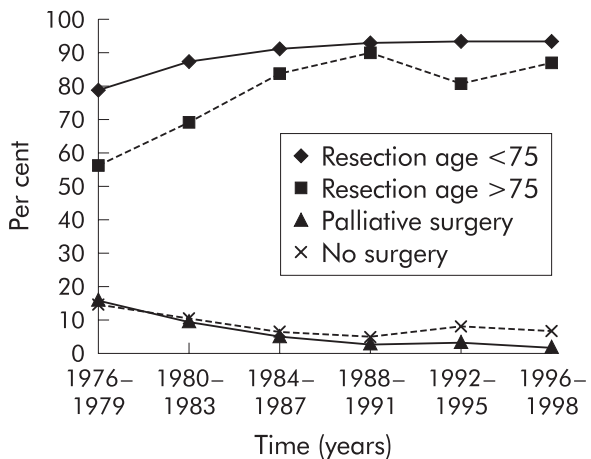

Figure 1 Time trends in surgical treatment.

stages; $p<0.0001)$. Of the patients presenting as an emergency, $67.9 \%$ underwent curative resection, $16.6 \%$ had palliative resection, and $14.0 \%$ had surgery but had no tumour resection $(\mathrm{p}<0.0001)$. Over time, the proportion of patients presenting as an emergency and who underwent an initial defunctioning colostomy with or without subsequent resection decreased significantly, in parallel with an increase in the proportion of patients who underwent a resection of the cancer with or without a stoma. The time trends in the emergency management are described in table 1 . The proportion of patients who presented as an emergency remained fairly stable over the 23 years of the study.

\section{Time trends in cancer resection}

Overall, $85.2 \%$ of the patients benefited from resection. The resection rate increased progressively from 69.3\% (1976$1979)$ to $91.9 \%$ (1988-1991) and then remained stable (the corresponding values for curative resections were $56.6 \%$ and $81.0 \%)$. Analysis by age group showed that this improvement was most impressive in the older age group with an increase from $56.4 \%$ to $90.5 \%$ compared with $78.9 \%$ to $93.2 \%$ for the those less than 75 years of age (fig 1).

Among the curative resections, $6.0 \%$ were endoscopic resections. The proportion of endoscopic procedures increased from $2.8 \%$ for the $1976-1979$ period to $8.7 \%$ for the $1992-1995$ period and subsequently decreased to $4.9 \%$ for the 1996-1998 period.

In a multivariate analysis, age, place of residence, metastatic status, and period of diagnosis were significant factors associated with the performance of tumour resection (table 2). Patients less than 75 years of age were 3.18 times more likely than older patients to undergo resection. Patients living in rural areas were less likely than patients living in the university hospital town and smaller towns to have a surgical procedure. The metastatic status decreased by 13 -fold the likelihood of such a procedure. Finally, the period of diagnosis was strongly associated with the performance of tumour resection, as the likelihood of a resection in the latest study period compared with the first rose by eightfold. Sex and location of the tumour were not significant factors. 
Table 2 Factors associated with resection procedures: multivariate analysis

\begin{tabular}{lcll}
\hline Factor & OR & $95 \% \mathrm{Cl}$ & $\mathrm{p} \mathrm{Value}$ \\
\hline $\begin{array}{l}\text { Metastatic status } \\
\text { Yes }\end{array}$ & 1 & & \\
$\quad$ No & 13.60 & $9.10-18.40$ & $<0.001$ \\
$\begin{array}{l}\text { Period of diagnosis } \\
\text { 1976-1979 }\end{array}$ & 1 & & \\
$1980-1983$ & 1.82 & $1.32-2.50$ & \\
$1984-1987$ & 4.06 & $2.83-5.81$ & \\
$1988-1991$ & 6.80 & $4.59-10.10$ & \\
$1992-1995$ & 5.76 & $3.96-8.39$ & \\
$1996-1998$ & 7.85 & $4.91-12.60$ & $<0.001$ \\
Age (y) & & & \\
$\geqslant 75$ & 1 & & \\
$<75$ & 3.18 & $2.52-4.00$ & $<0.001$ \\
Place of residence & & & \\
University town & 1 & & \\
Other urban & 1.10 & $0.80-1.51$ & \\
Rural & 0.78 & $0.61-0.99$ & 0.044 \\
\hline
\end{tabular}

Logistic regression carried out among patients treated by surgical or non-surgical procedures, excluding 21 cases with unknown tumour location.

*Probability for heterogeneity (likelihood ratio test).

OR, odds ratio; $95 \% \mathrm{Cl}, 95 \%$ confidence interval.

An important part of surgical treatment is whether or not a stoma is performed. Among the 2899 patients who underwent tumour resection, $157(5.4 \%)$ had a stoma procedure at the same time as cancer resection. Only 49 of those patients (33.1\%) had a reversal performed at a later date. A group of 111 patients first underwent a temporary stoma followed at a later stage by resection for cure combined with reversal in $51.3 \%$ of these cases. The proportion of patients, who had a stoma at the same time or before resection, who subsequently had a reversal increased from 33.3\% (1976-1979) to $47.8 \%$ (1996-1998). This surgical decision was mainly influenced by age as $29.3 \%$ of patients in the age group 75 years and above benefited from reversal compared with $50.3 \%$ in the younger age group $(\mathrm{p}<0.001)$. Over the 23 years of the study, the proportion of patients younger than 75 years of age undergoing reversal increased from $36.8 \%$ to $63.6 \%$ compared with a minimal increase in the older age group from $20.0 \%$ to $33.3 \%$. One hundred and forty five patients $(4.3 \%)$ had a stoma as the only treatment.

\section{Time trends in adjuvant chemotherapy}

Before 1989, adjuvant chemotherapy was an anecdotal treatment, with only 16 patients treated in total. The proportion of stage III patients treated with adjuvant chemotherapy rose progressively from $4.1 \%$ during the 1989-1990 period to $45.7 \%$ for the $1997-1998$ period. In the age group $>75$ years, the practice of adjuvant chemotherapy rose very slowly from none in $1989-1990$ to $25.9 \%$ in $1997-1998$ compared with $7.7 \%$ and $75.6 \%$ in the younger group for the same periods. An increase in the use of adjuvant chemotherapy was also noted for stage II tumours from $0 \%$ for the 1989-1990 period to $12.8 \%$ for the $1997-1998$ period.

Age, period of diagnosis, and stage at diagnosis were significant factors associated with the use of adjuvant chemotherapy in a multivariate analysis (table 3). Patients less than 75 years of age were 11 times more likely than older patients to receive adjuvant chemotherapy. The probability of adjuvant treatment for the 1990-1993 period was 7.1 times that of the earliest study period and the probability for the 1994-1997 period was 40 . The strongest factor associated with the use of adjuvant chemotherapy was stage at diagnosis, with a probability of 9.8 for stage II and a probability of 70 for stage III compared with stage I.
Table 3 Factors associated with adjuvant chemotherapy: multivariate analysis

\begin{tabular}{lccc}
\hline Factor & OR & \multicolumn{1}{c}{$95 \% \mathrm{Cl}$} & $\mathrm{p} \mathrm{Value}^{*}$ \\
\hline Stage at diagnosis & & & \\
$\quad$ Stage I & 1 & & \\
Stage II & 9.84 & $3.0-32.30$ & \\
$\quad$ Stage III & 70.30 & $21.5-230.0$ & $<0.0001$ \\
$\begin{array}{l}\text { Period of diagnosis } \\
\quad 1976-1989\end{array}$ & 1 & & \\
$1990-1993$ & 7.09 & $3.64-13.80$ & \\
$\quad 1994-1998$ & 39.90 & $22.0-72.40$ & $<0.0001$ \\
Age (y) & 1 & & \\
$\geqslant 75$ & 10.80 & $6.34-18.30$ & $<0.0001$ \\
$<75$ & & & \\
\hline
\end{tabular}

Logistic regression carried out among 2359 patients who underwent a curative resection of the tumour, excluding cases with unknown chemotherapy treatment, undetermined resection, no resection or palliative surgery, advanced, or unknown stages.

${ }^{*} \mathrm{p}$ for heterogeneity (likelihood ratio test).

$\mathrm{OR}$, odds ratio; $95 \% \mathrm{Cl}, 95 \%$ confidence interval.

\section{Time trends in palliative chemotherapy}

The use of palliative chemotherapy rose over the 23 years of the study from $4.0 \%$ in the $1976-1989$ period to $16.9 \%$ for the 1990-1993 period and finally to 34.5\% for the 1994-1998 period. Patients in the younger age group were more likely to receive palliative chemotherapy $(21.2 \% v 3.4 \%$ for those $\geqslant 75$ years of age; $\mathrm{p}<0.001)$. Palliative chemotherapy was used more often for those patients who underwent palliative resection $(18.6 \%)$, or a colostomy or exploratory laparotomy $(16.4 \%)$ as opposed to those who were not operated on $(6.3 \%$; $\mathrm{p}<0.001)$. There was no significant difference between the sexes or place of residence.

\section{Time trends in stage at diagnosis}

Over the 23 years of this study there was an increase in the proportion of patients diagnosed at an early stage. Indeed the percentage of stage I and II tumours increased from 39.6\% during $1976-1979$ to $56.5 \%$ during 1996-1998 (fig 2). This increase was correspondingly associated with a decrease in the proportion of patients with advanced stages (visceral metastasis, non-operated patients, and operated patients without resection of the tumour). The corresponding values for the same periods were $34.4 \%$ and $21.8 \%$. The proportion of stage III cancers remained fairly stable over the study period. There was no significant difference in stage at presentation between age groups or between the sexes. Of those presenting with stage I and II disease, $51.3 \%$ were less than 75 years of age and $48.6 \%$ were over 75 . The corresponding values for stage III disease were $23.9 \%$ and $24.3 \%$ and for advanced stages $24.4 \%$ and $26.8 \%(\mathrm{p}=0.4)$. Patients living in rural areas tended to present with more advanced disease compared with patients living in urban areas $(27.7 \% v 23.7 \% ; \mathrm{p}=0.048)$.

\section{Time trends in postoperative mortality}

Overall postoperative mortality decreased from 19.5\% for the 1976-1979 period to $7.3 \%$ for the 1996-1998 period. After curative resection the corresponding values were $14.8 \%$ and $7.3 \%$. Postoperative mortality after curative resection decreased even more noticeably in those younger than 75 years of age (from $13.7 \%$ for $1976-1979$ to $4.8 \%$ for $1996-1998$ ) whereas the corresponding values in the elderly were $16.8 \%$ and $9.9 \%$.

\section{Time trends in prognosis}

Prognosis improved over the study period: the five year relative survival rates were 33.0\% (1976-1979), 40.9\% (1980-1983), $52.5 \%$ (1984-1987), 58.9\% (1988-1991), and 55.3\% (19921995). Most noticeably there was a halving in the mortality 


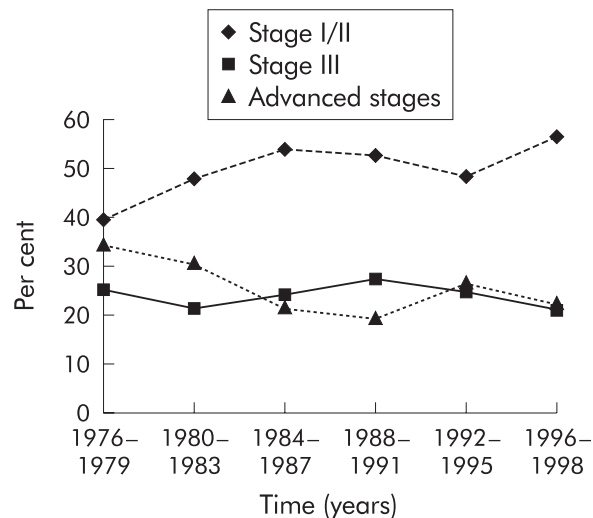

Figure 2 Time trends for the stage of tumours at the time of diagnosis.

Table 4 Relative mortality during different periods after diagnosis by year of diagnosis

\begin{tabular}{clc}
\hline & \multicolumn{2}{l}{ Mortality (\%) by period of diagnosis } \\
\cline { 2 - 3 } Year of diagnosis & 0-3 months & 4-60 months \\
\hline $1976-1979$ & 25.9 & 41.4 \\
$1981-1983$ & 21.4 & 37.6 \\
$1984-1987$ & 16.0 & 32.0 \\
$1988-1991$ & 13.3 & 27.8 \\
$1992-1995$ & 14.8 & 32.7 \\
$1996-1998$ & 12.9 & - \\
\hline
\end{tabular}

Relative mortality= 1-relative survival.

rate over the first three months (table 4). The decrease in mortality rate between four months and 60 months after diagnosis was less marked, decreasing from $41.1 \%$ (1976-1979) to $32.7 \%$ (1992-1995).

Factors with an impact on prognosis were analysed in a multivariate analysis. Stage at diagnosis was the most important prognosis factor (table 5). The period of diagnosis was also strongly related to survival. Other significant and independent prognostic factors associated with a poor prognosis were: presentation in an emergency context, age 75 years and over, and a tumour located in the right colon.

\section{DISCUSSION}

This study has demonstrated significant improvements in diagnostic and therapeutic approaches over a 23 year period, which has had an important impact on survival on this population. One of the major advantages of this type of analysis compared with hospital series analysis is that it is not affected by bias such as that inherent in case selection. Our study suggests several interesting trends in the management of colon cancer.

The first important trend concerns the rate of surgical resection. It increased over the first four study periods and then levelled out. It is now not far from optimum (resection rate is now over $90 \%$ ) and it is difficult to envisage a major improvement on this. Fewer patients undergo surgery in the older age group, as also reported in other studies. ${ }^{7-11}$ This difference between age groups is multifactorial; it may be because of later presentation, poor performance status, presence of comorbidities, or an expectation of a poorer outcome in elderly patients. However, the gap between the age groups is closing over time. The resection rate was also lower in rural than urban areas. There may be age related differences in the seeking of medical advice, recognition of symptoms, and referral patterns in primary care. Unsurprisingly, the
Table 5 Factors of prognostic significance in cancer of the colon: multivariate relative survival model

\begin{tabular}{|c|c|c|c|}
\hline \multirow[b]{2}{*}{ Factor } & \multicolumn{3}{|c|}{ Relative survival } \\
\hline & RR & $95 \% \mathrm{Cl}$ & p Value* \\
\hline \multicolumn{4}{|l|}{ Stage } \\
\hline Stage I & 1 & & $<0.0001$ \\
\hline Stage II & 2.79 & $1.75-4.46$ & \\
\hline Stage III & 8.36 & $5.30-13.19$ & \\
\hline Advanced & 30.35 & $19.30-47.72$ & \\
\hline \multicolumn{4}{|c|}{ Period of diagnosis } \\
\hline 1976-1979 & 1 & & $<0.0001$ \\
\hline 1980-1983 & 1.26 & $1.07-1.48$ & \\
\hline 1984-1987 & 0.98 & $0.83-1.17$ & \\
\hline 1988-1991 - - - & 0.82 & $0.69-0.98$ & \\
\hline 1992-1995 & 0.77 & $0.65-0.92$ & \\
\hline \multicolumn{4}{|c|}{ Emergency surgery } \\
\hline No & 1 & & $<0.0001$ \\
\hline Yes & 1.51 & $1.30-1.75$ & \\
\hline \multicolumn{4}{|l|}{ Age (y) } \\
\hline$<75$ & 1 & & 0.007 \\
\hline$\geqslant 75$ & 1.29 & $1.07-1.55$ & \\
\hline \multicolumn{4}{|l|}{ Location fumour } \\
\hline Left colon & 1 & & \\
\hline Right colon & 1.24 & $1.11-1.39$ & $<0.0001$ \\
\hline
\end{tabular}

3295 patients were studied, excluding cases with unknown status, tumour location, or stage at diagnosis.

*Probability for heterogeneity (likelihood ratio test).

$\mathrm{RR}$, relative risk of death; $95 \% \mathrm{Cl}, 95 \%$ confidence interval.

presence of visceral metastasis was another factor significantly associated with the non-performance of tumour resection. The rate of surgical resection varies widely within Europe. ${ }^{11}$ The resection rate in the Côte-d'Or area is among the highest in Europe, similar to those reported in the Netherlands, Italy, and other French areas. It is higher than those reported in Spain, the UK, and Poland. It has been suggested that difference in stage at diagnosis is the main explanation for these variations. ${ }^{12}$ This study highlights the rather high proportion of patients with a colon cancer who had a colostomy. Even in the most recent study period, only half of the patients who underwent a colostomy and who were resected for cure had a reversal at a later date. A survey done in France in seven different areas has already underlined the fact that a stoma was not as infrequent in the treatment of colon cancer: $17.4 \%$ in 1990 and $16.8 \%$ in $1995 .{ }^{13}$

The second important trend noted was an increase in the proportion of patients diagnosed at an early stage and a resultant decrease in the proportion of patients with advanced stages. This is likely to be multifactorial, again due to patients seeking medical advice more rapidly and to more rapid referral for investigations by general practitioners. We also showed that there was no difference in stage at presentation between age groups, unlike other studies concluding that elderly patients are more likely to present with later stage disease than their younger counterparts. ${ }^{14}{ }^{15}$

The third important trend concerns the increasing use of chemotherapy for the treatment of colon cancer. Adjuvant chemotherapy started to be used more widely when scientific evidence of effectiveness of this treatment for TNM stage III colon cancer became available from 1990. ${ }^{16}$ However, the development of the use of adjuvant chemotherapy between 1989 and 1998 has been relatively slow and much progress is still possible. Our study showed that there was a delay between the publication of scientific evidence about the effectiveness of adjuvant chemotherapy for the treatment of stage III colon cancer and the implementation of this treatment in the Côte-d'Or region. Such information can only be provided by this type of population based study. The proportion of patients with stage III disease receiving adjuvant chemotherapy was $13.5 \%$ for the 1989-1993 period in this study, well 
below the value of $43 \%$ for those receiving this treatment in the USA, according to the American National Cancer Data Base Report on colon cancer for the period 1988-1993. ${ }^{17}$ Differences in the practice of adjuvant chemotherapy are seen within France. In a special survey conducted by seven French cancer registries in 1995, the proportion of patients under 80 years receiving adjuvant chemotherapy for stage III colon cancer varied between $38.1 \%$ and $50.0 \%{ }^{13}$ Age played an important role in the use of adjuvant chemotherapy. Our study showed that patients who are mostly being offered adjuvant chemotherapy are those in the younger age group with stage III colon cancer, $75 \%$ of these patients being treated over the last study period. Chemotherapy was much less likely to be used for older patients. Considering the increase in life expectancy and the relatively low toxicity of adjuvant chemotherapy used in colon cancer, a larger proportion of the elderly population could benefit from this treatment. ${ }^{18}$ The role of adjuvant chemotherapy for the treatment of stage II colon cancer is still controversial. A meta-analysis of five randomised studies testing the value of 5 fluorouracil and folinic acid for stage II colon cancers showed no improvement in overall survival or disease free survival. ${ }^{19}$ Therefore, there should be no indication for adjuvant chemotherapy for stage II colon cancer outside clinical trials. However, $12.8 \%$ of patients with stage II colon cancer received adjuvant chemotherapy for the last study period. The proportion of patients with stage II colon cancer treated with adjuvant chemotherapy in the study conducted by seven French cancer registries was, on average, $23.9 \%$, with variations between $10.0 \%$ and $35.4 \%$ according to the area. ${ }^{13}$

The most striking trend lies in the major improvement in survival over the 23 years of the study period. This finding is consistent with the results of the Eurocare study comparing colon cancer survival in 17 European countries. It showed a general improvement in five year survival rates from $40 \%$ to $48 \%$ over the period $1978-1989 .{ }^{20}$ However, there were important differences in survival of colorectal cancer patients between the different countries within Europe during the period 1978-1989. France ranks among the countries with the highest survival in Europe together with the Nordic countries (Denmark excluded), the Netherlands, Switzerland, and Austria. The UK, Denmark, and the Eastern European countries were characterised by a low survival. Longer survival can be explained by earlier diagnosis, better treatment, and improved perioperative care. If survival is more affected in the three months after diagnosis it is more likely to be due to a decrease in postoperative mortality and early stage at diagnosis. ${ }^{21}{ }^{22}$ Our study demonstrated an immediate reduction in mortality at three months and also an improvement in long term survival. This implies that postoperative mortality was not the only factor responsible for better prognosis and that early stage at diagnosis and improved medical and surgical care also have important roles to play. In a recent high resolution EUROCARE study ${ }^{12}$ it was demonstrated that after appropriate correction for stage at diagnosis, only one of the 11 countries (Poland) had a significant poorer survival. This shows that the wide differences across Europe in colorectal cancer survival depend to a large extent on differences in stage at diagnosis.

In conclusion, early detection and the optimum combination of surgery and adjuvant chemotherapy can make a significant impact on the outcome of colon cancer. An optimum has probably been reached for tumour resection and reduction in operative mortality but progress can still be made, particularly regarding the use of adjuvant chemotherapy in the older age group and early detection of colon cancer.

\section{ACKNOWLEDGEMENTS}

We would like to thank Dr KL Finn for his work on the draft of this article and the Ligue Bourguignonne Contre le Cancer for financial support.

\section{Authors' affiliations}

C Faivre-Finn, A-M Bouvier-Benhamiche, J M Phelip, S Manfredi, V

Dancourt, J Faivre, Registre Bourguignon des Cancers Digestifs (INSERM EPI 106), Faculté de Medecine, BP 87900, 21079 Dijon, France

\section{REFERENCES}

1 Menegoz F, Black RJ, Arveux P, et al. Cancer incidence and mortality in France in 1975-1995. Eur J Cancer Prev 1997:6:442-66.

2 Colemann MP, Esteve J, Damiecki P, et al. Trends in cancer incidence and mortality. IARC scientific publication No. 121. Lyon: International Agency for Research on Cancer, 1993

3 WHO. International classification of diseases for Oncology, 10th revision. Geneva: World Health Organization, 1990.

4 Sobin L, Wittekind C. Colon and rectum. In: Hermanek P, Hutter RVP, Sobin LH, et al, eds. TNM Atlas, International Union Against Cancer, 4 th edn. Berlin: Springer-Verlag, 1997.

5 Dixon WJ, Brown MB, Engelman L, et al. BMDP statistical software. Los Angeles: University of California Press, 1981.

6 Esteve J, Benhamou E, Croasdale M, et al. Relative survival and the estimation of net survival: elements for further discussion. Stat Med 1990;9:529-38.

7 Waldron RP, Donovan IA, Drumm J, et al. Emergency presentation and mortality from colorectal cancer in the elderly. Br J Surg 1986;73:214-16.

8 Samet J, Hunt WC, Key C, et al. Choice of cancer therapy varies with age of patient. JAMA 1986;255:3385-90.

9 Irvin TT. Prognosis of colorectal cancer in the elderly. Br J Surg 1988:75:419-21.

10 Iscoe N, To P, Gort E, et al. Cancer surgery in the elderly. Ann Oncol 1998;9:109

11 Gatta G, Sant M, Coebergh JW, et al. Substantial variation in therapy for colorectal cancer across Europe: EUROCARE analysis of cancer registry data for 1987. Eur J Cancer 1996;32:831-5.

12 Gatta G, Capocaccia R, Sant M, et al. Understanding variations in survival for colorectal cancer in Europe: a EUROCARE high resolution study. Gut 2000;47:533-8.

13 Maurel J, Pottier D, Grosclaude P, et al. Prise en charge thérapeutique du cancer colique en France. Gastroenterol Clin Biol 1998;22:90-6.

14 Colorectal Cancer Collaborative Group. Surgery for colorectal cancer in elderly patients: a systematic review. Lancet 2000;356:968-74

15 Havlik RJ, Yancik R, Long S, et al. The National Institute on Aging and the National Cancer Institute SEER collaborative study on comorbidity and early diagnosis of cancer in the elderly. Cancer 1994;74:2101-6.

16 Moertel CG, Fleming TR, Macdonald JS, et al. Levamisole and fluoro-uracil for adjuvant therapy of resected colon cancer. N Engl J Med 1990;322:352-8

17 Milburn J, McGinnis LS, Steele GD, et al. The National Cancer Data Base Report on Colon Cancer. Cancer 1996:78:918-26.

18 O'Connell MJ, Mailliard JA, Khan M, et al. Controlled trial of fluorouracil and low dose leucovorin given for 6 months as postoperative adjuvant therapy for colon cancer. J Clin Oncol 1997;15:246-50.

19 International multicentre pooled analysis of B2 colon cancer trials (IMPACT B2) investigators: efficacy of adjuvant fluorouracil and folinic acid in B2 colon cancer. J Clin Oncol 1999;17:1356-63.

20 Gatta G, Faivre J, Capocaccia R, et al. Survival of colorectal cancer patients in Europe during the period 1978-1989. Eur J Cancer 1998:34:2176-83.

21 Sant M, Capocaccia R, Verdecchia A, et al. Comparisons of colon-cancer survival among European countries: the EUROCARE study. Int J Cancer 1995;63:43-8.

22 Enblad P, Adami HO, Bergtröm R, et al. Improved survival of patients with cancer of the colon and rectum? J Nat Cancer Inst 1988;80:586-91. 